\title{
Sistema de informação em enfermagem: interação do conhecimento tácito-explícito
}

\author{
Information system in nursing: interacion of tacit-explicit knowledge \\ Sistema de información en enfermería: interacción del conocimiento tacito-explicito
}

\author{
Sérgio Ribeiro dos Santos \\ Enfermeiro. PhD. Professor do Departamento \\ de Enfermagem - DEMCA/CCS/UFPB \\ srsantos@jpa.neoline.com.br
}

\section{RESUMO}

O presente artigo tem por objetivo traçar algumas considerações teóricas e conceituais sobre sistema de informação em enfermagem, buscando situar o conhecimento baseado nas evidências da prática clínica para construir um modelo de sistema integrado às estruturas conceituais, formadas pela combinação de três ciências: informação, computação e enfermagem. Esse conhecimento pode descrever e explicar sistematicamente os fenômenos necessários para desenvolver um compreensivo sistema de informação que contribua para a melhoria dos registros de enfermagem e consolide os mecanismos para prover medidas básicas de custos, qualidade, acesso ao cuidado do paciente e resultado deste cuidado.

Descritores: Sistema de informação; Enfermagem; Conhecimento.

\section{ABSTRACT}

The present article aims to trace some theoretical and conceptual considerations on information systems in nursing, seeking to point out the knowledge based on the clinical practice evidences to construct a model of system integrated to the conceptual structures, formed by the combination of three sciences: information, computing and nursing. This knowledge can systematically describe and explain the necessary phenomena to develop a comprehensive information system that contribute for nursing records improvement and to consolidate a mechanism to provide basic measuring of costs, quality, patient access to care, and results of this care. Descriptors: Information systems; Nursing; Knowledge.

\section{RESUMEN}

El presente artículo tiene por objetivo trazar algunas consideraciones teóricas y conceptuales sobre sistema de información en enfermería, buscando situar el conocimiento basado en las evidencias de la práctica clínica para construír un modelo de sistema integrando las estructuras conceptuales, formadas por la combinación de tres ciencias: información, computación y enfermería. Ese conocimiento puede describir y explicar sistemáticamente los fenómenos necesarios para desarrollar un comprensivo sistema de información que contribuya para la mejora de los registros de enfermería y consolide los mecanismo para proveer medidas básicas de costos, calidad, acceso al cuidado del paciente y resultado de este cuidado.

Descriptores: Sistemas de información; Enfermería; Conocimiento.

Santos SR. Sistema de informação em enfermagem: interação do conhecimento tácito-explícito. Rev Bras Enferm 2005 jan-fev; 58(1):100-4.

\section{INTRODUÇÃO}

A utilização de modelos conceituais (conhecimento explícito) sem levar em consideração as necessidades da prática (conhecimento tácito) favorece o insucesso ou fracasso na utilização dos sistemas. Vários modelos conceituais têm sido propostos nos manuais de informática em enfermagem. No entanto, poucos apresentam um resultado custo-benefício satisfatório.

Este estudo está estruturado em três tópicos. No primeiro, discute-se sucintamente sobre as dimensões do conhecimento. No segundo, são apresentados os elementos integrados ao desenvolvimento de sistemas de informação, onde se localiza o tema proposto. Finalmente, no terceiro, busca-se integrar o conhecimento tácito com o conhecimento explícito, objetivando-se propor um modelo de sistema que represente uma alternativa para o desenvolvimento de software aplicado à enfermagem.

\section{DIMENSÕES DO CONHECIMENTO TÁCITO E EXPLÍCITO}

A compreensão do conhecimento tácito e explícito em enfermagem como elementos indispensáveis para o desenvolvimento de sistemas de informação é fundamental para o êxito de um software. Os pesquisadores de enfermagem têm se esforçado no sentido de desenvolver sistemas de informação que focalizem a importância da padronização de uma linguagem comum. Nessa perspectiva, colher informações, a partir do ambiente de trabalho do enfermeiro, torna-se imprescindível para que se possa ajustar esse paradigma à tecnologia da informação. 
Sob essa ótica, a padronização com vista ao desenvolvimento de um sistema de informação tem suas limitações, porque ela surge de fora para dentro, com o intuito de resolver problemas operacionais que não se adaptam à dinâmica do ambiente hospitalar. É oportuno lembrar que os sistemas de classificação em enfermagem têm sido enfatizados como uma ferramenta destinada a melhorar o conhecimento teóricoprático da enfermagem. A diferença entre o conhecimento formal ou explícito (teoria) e o conhecimento informal ou tácito (prática) precisa ser considerada, não apenas para efeito de desenvolvimento do sistema de informação, mas principalmente com o propósito de melhorar a qualidade da informação gerenciada pela enfermagem.

Convém esclarecer que a distinção entre conhecimento tácito e conhecimento explícito, com base na dimensão epistemológica, já vem sendo estudada por Michael Polanyi desde a década de 1960. O conhecimento tácito é pessoal, específico ao contexto e, assim, difícil de ser formulado e comunicado. É aquele conhecimento que as pessoas possuem, mas não está escrito em lugar nenhum, residindo apenas em suas cabeças. Já o conhecimento explícito é transmitido em linguagem formal e sistemática; é aquele que está registrado de alguma forma e, assim, disponível para as demais pessoas.

Em tese, podemos evidenciar, no dia-a-dia da prática de enfermagem, a complexidade de conhecimentos que envolvem as ações de enfermagem, observando-se que seus profissionais podem saber muito mais do que são capazes de dizer e registrar. Porém, esse conhecimento é um recurso que precisa ser gerenciado para atingir plenamente os objetivos organizacionais.

O conhecimento em enfermagem é um conjunto constituído por experiências, valores, informações contextuais e avaliação de novas experiências e informações. Nessa perspectiva, é visto como algo inseparável nas pessoas. Para a enfermagem, o conhecimento se encontra não apenas nos documentos (prontuários, livros de registro, manuais etc.) ou nas bases de dados e sistemas de informação, mas também nos processos, na prática e na experiência acumulada pelo pessoal de enfermagem.

O conhecimento apreendido pelos enfermeiros ocorre de diversas formas: por comparação, pela experimentação, por associação com outros conhecimentos e por intermédio de outros profissionais da área de saúde (médicos, nutricionistas, farmacêuticos etc.). Na realidade, 0 conhecimento é adquirido no dia-a-dia do trabalho da enfermagem, sendo transmitido de pessoa para pessoa, através de meios estruturados (vídeos, livros, documentos, Web etc.) e não-estruturados. Significa dizer que os enfermeiros adquirem conhecimento das pessoas que já o têm, ou seja, é o aprendizado interpessoal e o compartilhamento de experiências e idéias.

Na prática, o pessoal de enfermagem cria, em sua mente, um modelo do ambiente hospitalar em que está inserido, incluindo elementos cognitivos e técnicos. O elemento cognitivo pode ser representado por esquemas, paradigmas, perspectivas, crenças e pontos de vista que ajudam a compreender o mundo em sua volta, enquanto o elemento técnico inclui know-how (conhecimento das técnicas e habilidades de enfermagem). Esses elementos constituem o conhecimento tácito, o qual permite a visualização da realidade, ou seja, "o que é", e a percepção "do que deveria ser". A compreensão desses elementos constitui o fator-chave para o desenvolvimento de um sistema de informação fundamentado na interação teoria-prática.

Portanto, não se pode desvincular o conhecimento tácito do explícito em enfermagem para o desenvolvimento de um sistema de informação, porque eles são elementos que não se separam, mas se complementam. Assim, teoria e prática interagem e cambiam atividades que podem ser executadas de forma consistente, sistematizada e lógica. Pode-se afirmar que o modelo de sistema de informação em enfermagem deve estar fundamentado numa conjuntura crítica em que o conhecimento surge e se expande a cada dia, através da inter-relação entre a teoria e a prática. Essa interação envolve fatores subjetivos e objetivos veiculados através do meio social. A cognição humana é um processo dedutivo de indivíduos, mas um indivíduo nunca está isolado da interação social quando desenvolve seus conhecimentos. Assim, através do processo de conversão social, a teoria e a prática se expandem, tanto em termos de qualidade, quanto de quantidade ${ }^{(1)}$.

Por outro lado, existe a compreensão de que o conhecimento da prática é um recurso imenso, mas não aproveitado. Em conseqüência, poucas pesquisas têm sido desenvolvidas na comunidade acadêmica buscando identificar o motivo por que esses profissionais não usam a pesquisa baseada no conhecimento da prática. Embora o enfermeiro tenha à sua disposição uma estrutura hospitalar para criar novos conhecimentos, esse saber não é codificado ou publicado. Além disso, nem sempre é possível fazer uma reflexão ou estabelecer uma discussão no próprio ambiente de trabalho ${ }^{(2,3)}$.

Em conseqüência, o conhecimento da prática torna-se uma "dor de cabeça metodológica" pela escassez de pesquisas que relatem essa experiência, embora muitos aspectos da enfermagem possam ser pesquisados, através da participação dos enfermeiros assistenciais. Em vista disso, questões epistemológicas envolvendo o objeto do conhecimento em enfermagem e sua filosofia devem ser bem analisadas, a fim de que seja possível dar respostas às indagações sobre a profissão como uma prática, uma ciência e uma arte.

Portanto, a criação do conhecimento em enfermagem é uma interação constante e dinâmica entre teoria e prática. É a partir dessa compreensão que se pode moldar um sistema de informação baseado nas diversas variáveis que compõem a realidade da enfermagem. Somente com a socialização do conhecimento, que começa no campo da interação, é possível compartilhar experiências e paradigmas entre os membros da equipe de enfermagem. Para externar suas opiniões, os membros da equipe devem fazer uso do diálogo ou da reflexão coletiva, onde se discutem questões relacionadas ao trabalho, contribuindo para estabelecer as bases do conhecimento prático ainda inexplorado. Quando esse conhecimento é integrado ao conhecimento já existente, forma-se um novo produto.

\section{INTEGRAÇÃO DOS ELEMENTOS DE UM SISTEMA DE INFORMAÇÃO}

Em tese, a relação teoria-prática em enfermagem no sistema de informação nos conduz a situações que se inter-relacionam, dentro da visão de mundo dos enfermeiros, vivenciado a partir das experiências desses profissionais. Assim sendo, partiu-se da compreensão de que esse processo não deve ser dissociado, mas integrado ao seguinte contexto: organizacional; conhecimento (teoria-prática) e o sistema de informação, conforme mostra a figura 1.

\section{a) $O$ contexto organizacional}

Uma organização deve ser interpretada como um sistema complexo, com inúmeros subsistemas interdependentes. No macroambiente organizacional, existe um conjunto de pessoas que formam uma sociedade onde ocorrem as interações. Nessa sociedade, os enfermeiros estão interagindo, trocando informações, interpretando, definindo e alterando a direção dos atos uns dos outros. Portanto, a organização não se constitui como uma entidade isolada, principalmente, no hospital.

Nesse ambiente, os enfermeiros formam um corpo social que está constantemente interagindo, construindo e reconstruindo um processo social, ao lidarem com a interdisciplinaridade, em que os significados são também socializados. Percebe-se que diante da complexidade da organização hospitalar, os enfermeiros interagem uns com os outros. Esta visão gera a impressão de que a idéia de se implantar um sistema de informação é problemática, uma vez que a informação está fundamentada no significado.

Na perspectiva organizacional para desenvolver um sistema de informação em enfermagem, os enfermeiros atribuem intersubjetivamente significados ao ambiente onde estão inseridos e são capazes de construir uma visão de informação concernente à sua realidade social. Tais manifestações podem ser utilizadas para interpretar e dar um significado a algumas complexidades do mundo vivenciado pelos enfermeiros.

Desta forma, a organização passa de objeto físico a objeto social, onde o mundo é interpretado de forma que reconheça como legítimas as ações participativas que estabelecem normas e padrões sociais 


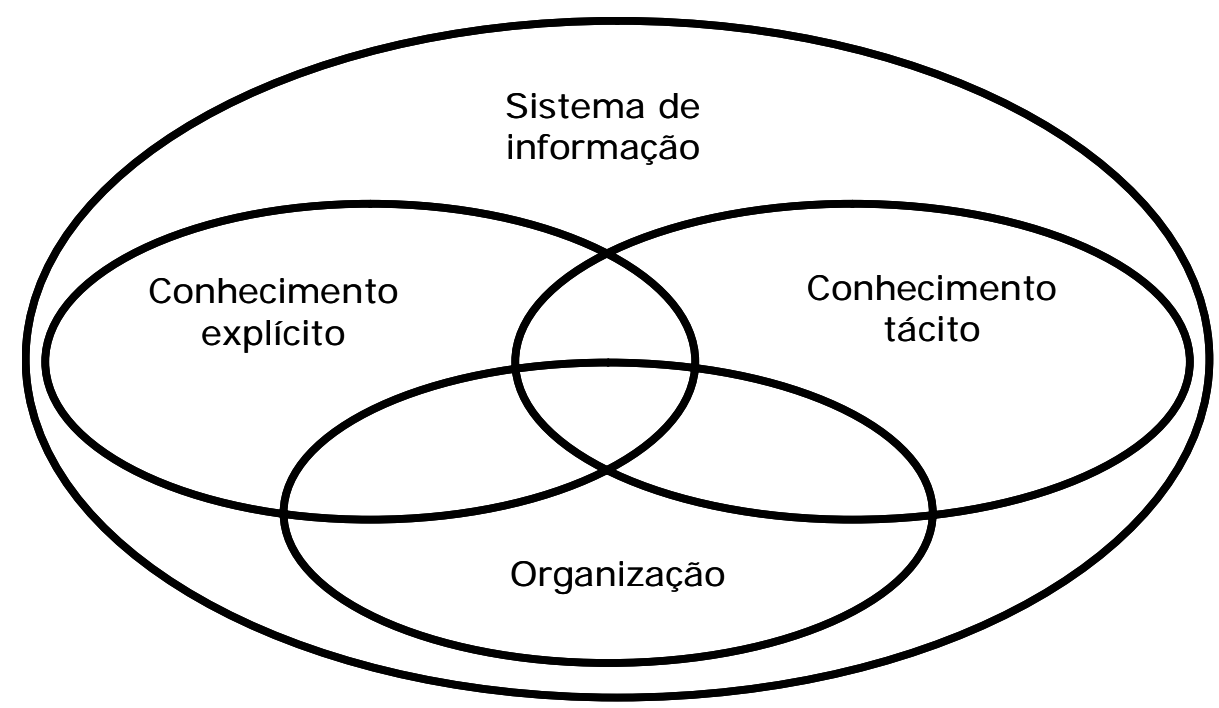

Figura 1. Elementos integrados ao desenvolvimento de sistemas de informação.

compartilhados $^{(4)}$. Portanto, não se pode discutir sistemas de informação sem enfocar o contexto organizacional.

\section{b) A dimensão do conhecimento (teoria-prática)}

No hospital, os enfermeiros assumem muitas atribuições e absorvem um complexo conhecimento de trabalho. Antes de analisar-se essa questão, é preciso fazer a seguinte observação: o conhecimento diz respeito a crenças e compromissos, sendo uma função de atitude, perspectiva ou intenção específica; o conhecimento está relacionado à ação, na medida em que tem algum objetivo e significado, sendo relacional e específico ao contexto(1).

A complexidade do conhecimento em enfermagem reflete-se nas tarefas associadas, como o processamento da informação, nas quais se incluem a acumulação de dados, o uso de informação, a aplicação do conhecimento na prática clínica e os novos conhecimentos em geral. Assim, para que possam desempenhar efetivamente seu papel, os enfermeiros se apóiam no ambiente da prática clínica para interpretar os eventos ou objetos, tornando visíveis os significados antes invisíveis na sua função de aplicadores do conhecimento. Por isso, o conhecimento em enfermagem está essencialmente relacionado com as ações de enfermagem.

O conhecimento em enfermagem é criado pelos próprios enfermeiros e depende do contexto organizacional em que está inserido. Entretanto, temos observado uma lacuna entre a teoria e a prática clínica. A natureza dessa lacuna tem sido uma grande preocupação para os enfermeiros assistenciais, pesquisadores e profissionais engajados em educação e treinamento de pessoal. A questão é descobrir em que nível a prática reflete a teoria e como minimizar os problemas associados com a disfunção da relação teoria-prática.

Os enfermeiros trazem consigo um vasto conhecimento formal e vão, ao longo da vivência prática, adquirindo conhecimentos, a partir de suas próprias experiências, de forma que eles podem saber mais do que admitem. $O$ conhecimento tácito e o explícito não são totalmente separados, mas mutuamente complementares. Em tese, a interação teoria e prática em enfermagem pode ser considerada um caminho para desenvolver sistemas de informação, com base nas seguintes suposições:

- a teoria é um recurso que deve ser usado ou integrado dentro da prática;

- a prática expressa um conhecimento potencial para completar ou contribuir para completar a integração com a teoria.

Na interação teoria e prática, a teoria deve estar voltada diretamente para a prática, enquanto as ações da prática têm a função de testar a teoria. A excelência da prática está embutida na prática em si e a teoria é derivada da prática, que é um ato social e não meramente uma ciência aplicada ${ }^{(5)}$. Portanto, a caracterização do conhecimento em enfermagem é um processo de visão que tem por base uma epistemologia interpretativa, em que o conhecimento explícito é necessário para descrever, interpretar e explicar a prática (conhecimento tácito).

\section{d) Sistema de informação}

O sistema de informação em enfermagem deve ser entendido como um processo complexo que envolve estudos técnicos, organizacionais, comportamentais e ambientais. A integração do sistema com a prática e o processo de enfermagem envolvem muitos fatores como educação, mudanças de atitude, cultura organizacional, padronização da linguagem e prática sistematizada. Portanto, o sistema de informação em enfermagem não é um software ou um hardware, na medida em que envolve pessoas, estrutura organizacional e processos que permitem a coleta de dados, o processamento e o uso racional da informação(6).

Nesta perspectiva, os enfermeiros e analistas de sistemas, ao tentarem desenvolver um software usando a metodologia tradicional, ignoram o complexo contexto organizacional e social que os envolve. Na realidade, os enfermeiros, assim como os profissionais de saúde e as pessoas em geral, têm pouco conhecimento em tecnologia e sistemas de informação e acreditam que a tecnologia, por si só, é a solução para todos os problemas. Em conseqüência, há uma má interpretação na definição de tecnologia, que é vista, geralmente, como o "computador" e seus programas.

A Organização Pan-Americana de Saúde define tecnologia da informação como um conjunto de conhecimentos técnicos e práticos, que tem o objetivo de favorecer sua aplicação no atendimento dos usuários. Envolve a criação, direção e suprimento de recursos tecnológicos (hardware, software e comunicação), necessários para 0 desenvolvimento e operação de aplicações documentais de uma organização(7).

A tecnologia está voltada para um mercado onde as pessoas são convidadas a adaptar-se a ela, em vez de procurarem outras formas que podem ser adequadas. Nessa perspectiva, a tecnologia deve ir ao encontro das necessidades das pessoas, beneficiando-as no seu bemestar e na qualidade de vida. Essa abordagem sugere que a tecnologia usada no desenvolvimento do sistema de informação deve ter uma configuração sociotécnica. Isso pressupõe um enfoque tanto empíricoanalítico como fenomenológico-hermenêutico, além de uma visão de mundo socialmente determinada pela experiência vivenciada na prática $^{(8)}$.

Assim, para que se possa estabelecer a interação teoria e prática 
no sistema de informação em enfermagem, é indispensável a participação dos profissionais de enfermagem no processo de desenho do sistema. Para tanto, eles devem considerar o que está acontecendo na prática, em termos de definição, interpretação, significado, conhecimentos baseados no trabalho, objetos, comunicação, símbolos, processos e comportamento social. Vale salientar que a interação da organização com o conhecimento (tácito/explícito) e o sistema de informação supõe a captura e o processamento de dados de enfermagem com ampla densidade de detalhes. Isso permite 0 estabelecimento de um registro longitudinal do paciente, que pode ser desenvolvido através de sistemas computadorizados e do registro eletrônico.

\section{MODELO COMPUTACIONAL DE SISTEMA DE INFORMAÇÃO EM ENFERMAGEM}

Vários modelos de sistemas de informação vêm sendo propostos para descrever diferentes aspectos do registro de enfermagem, mas nenhum foi desenvolvido com a intenção de atender as necessidades da práxis assistencial, em termos de eficiência e eficácia. O resultado é a baixa efetividade nos registros manuais e a subutilização dos registros informatizados, isso quando existe tecnologia disponível.

O processo de enfermagem, como parte do sistema de informação e como instrumento que direciona os cuidados ao paciente, não tem sua aplicabilidade na prática de enfermagem tal qual se ensina nos cursos de graduação em enfermagem. Essa deficiência ocorre porque o corpo de conhecimento da enfermagem apresenta várias estruturas conceituais e métodos para se pôr em prática nas mais diversas situações, o que exige do profissional de enfermagem características cognitivas e afetivas como fatores basilares a considerar.

Vale salientar que o registro ajuda a construir a base de conhecimentos (teórico e prático) para o desenvolvimento científico da enfermagem e a formação profissional. A documentação em si torna visível o trabalho invisível da enfermagem diante do sistema de informação hospitalar e do registro médico-eletrônico. Na realidade, somente o trabalho que é visível pode verdadeiramente ser identificado como valioso $0^{(9)}$.

Por outro lado, essa necessidade de visibilidade tem provocado profundas reflexões acadêmicas sobre a natureza do trabalho da enfermagem. O problema é que os modelos são desenvolvidos sob vários níveis de abstrações. Assim, oferecem caminhos para organizar e categorizar os fenômenos de enfermagem, tais como demonstrado pela North American Nursing Diagnosis Association - NANDA, Sistema Omaha, Classificação das Intervenções de Enfermagem - NIC e Classificação dos Resultados de Enfermagem - NOC.

A esse respeito, pode-se indagar: Por que, diante de tantos modelos explorados na literatura, o registro sistematizado das informações em enfermagem, mesmo no nível mais básico de informação, não funciona com eficiência e eficácia? Várias razões podem ser apontadas para explicar essa situação. Uma delas é que o empregador, os fornecedores e a política da organização hospitalar requerem objetividade nas informações. Ou seja, quem tem o poder de decisão precisa dispor de informações com base científica e objetiva sobre o cuidado de saúde, a fim de promover acesso ao serviço, alocar recursos, bem como determinar os custos e a qualidade da assistência de enfermagem.

Outra razão reside no fato de que o registro de informação em enfermagem evidencia a responsabilidade do processo assistencial e os resultados do cuidado. Daí a importância do registro, por ser ele 0 relato da qualidade e dos custos produzidos pela enfermagem. A falta de registro sistematizado ou a deficiência nas informações compromete o nível de qualidade desejada.

Finalmente, os modelos teóricos de registro de enfermagem para 0 sistema de informação precisam responder questões relacionadas à prática e ao conhecimento tácito. Precisam também identificar a "melhor" prática e desenvolver um padrão de vocabulário ou linguagem comum para 0 plano de cuidados e apoio à decisão que determine 0 que a enfermagem faz. Além disso, devem aumentar a autonomia e a satisfação no trabalho, bem como diminuir o aspecto negativo do ambiente da prática.

Um modelo de sistema que procure satisfazer essas exigências dimensão do conhecimento em enfermagem - requer um vocabulário derivado do conhecimento tácito com a interface do conhecimento explícito, de modo que reflita uma integração e classificação desse conhecimento. Em outras palavras, a prática individual (conhecimento tácito) é usada para construir informações e conhecimentos que apóiam a tomada de decisão da equipe de enfermagem.

Nenhum, dentre os vários modelos propostos de informática em enfermagem, possui uma estrutura organizacional guiada por uma teoria de desenvolvimento de sistemas de informação, porque tais modelos não incluem todos os componentes dos metaparadigmas em enfermagem, mas somente alguns contextos.

Assim, um modelo de sistema de informação em enfermagem pode ser construído com base na dinâmica de quatro componentes, em um sistema complexo de cuidado. Esse modelo também incorpora metaparadigmas em enfermagem, tais como: a organização, que retrata o ambiente de trabalho; o conhecimento, nos níveis tácito e explícito; as intervenções ou prescrições de enfermagem, à luz desse conhecimento; os resultados ou avaliação do estado de saúde.

A figura 2 apresenta o outline de um modelo de sistema de informação em enfermagem para melhor compreender os níveis de abstração desta estrutura.

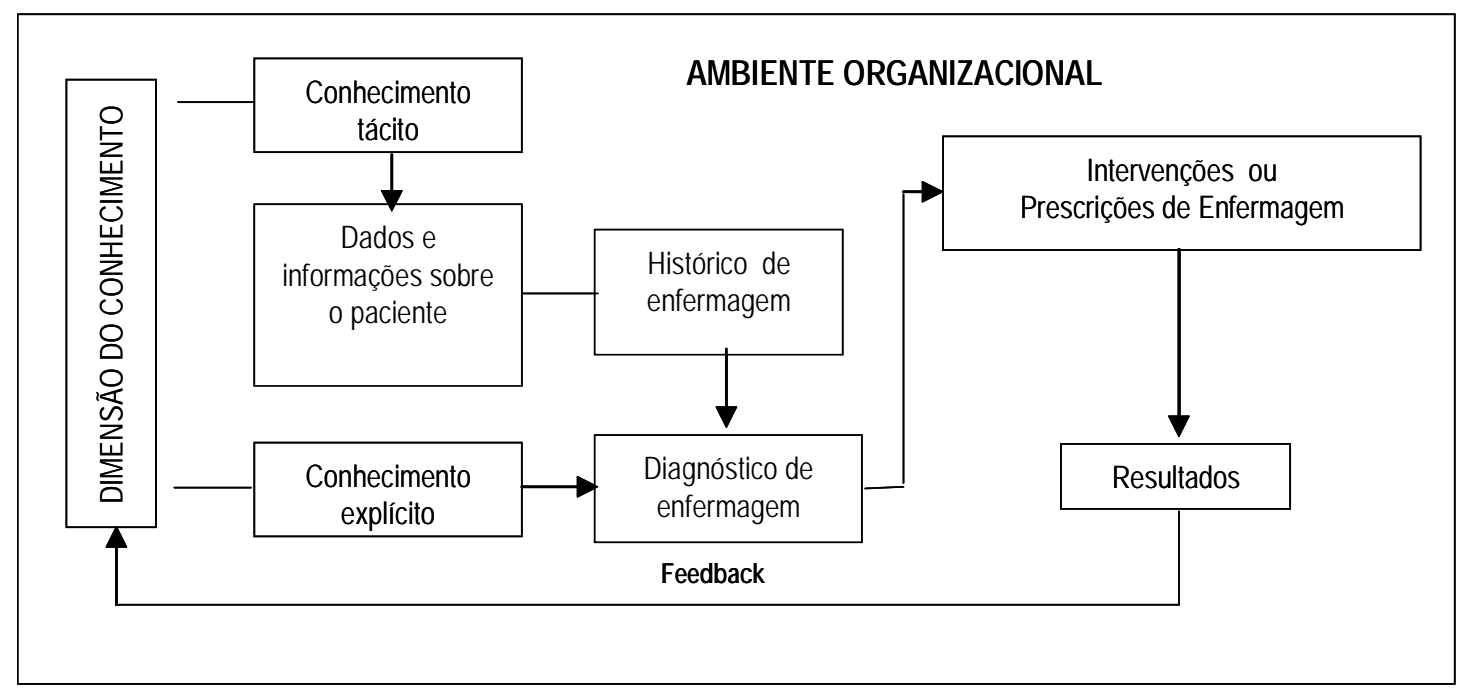

Figura 2. Modelo de sistema de informação em enfermagem. 


\section{CONSIDERAÇÕES FINAIS}

Defendemos a idéia de se desenvolver um sistema de informação em enfermagem, estruturado a partir da integração entre o conhecimento tácito e o conhecimento explícito. Esse sistema deve estar inserido no ambiente organizacional, de modo a facilitar o registro de enfermagem e agilizar o processo de trabalho, através da integração de um vocabulário, classificação e linguagem aceitos universalmente.

Essas estratégias são necessárias porque a tecnologia da informação requer uniformidade, precisão e dados sobre o cuidado do paciente, permitindo análises que proporcionem aumento na qualidade do cuidado. Assim, uma ferramenta eletrônica de suporte à gestão do serviço de enfermagem pode ser usada:

\section{REFERÊNCIAS}

1. Nonaka I, Takeuchi H. Criação de conhecimento na empresa: como as empresas japonesas geram a dinâmica da inovação. Rio de Janeiro (RJ): Campus; 1997.

2. Meerabeau L. Tacit nursing knowledge: an untapped resource or a methodological headache? J Adv Nurs 1992 jan;17(1):108-12.

3. Assad LG, Viana LO. Saberes práticos na formação do enfermeiro. Rev Bras Enferm 2003 jan-fev;56(1):44-7.

4. Checkland $P$, Holwell $S$. Information, systems and information systems. Chichester: Wiley \& Sons; 1998.

5. Benner $P$, Wrubel J. The primacy of caring. San Francisco (CA): Addinson-Wesley; 1989.
- com link da literatura em enfermagem para extrair informações do conhecimento explícito;

- com link para os sistemas formais: NIC, NOC, NANDA, Omaha e o sistema de classificação em saúde domiciliar;

- com inclusão de todo conhecimento tácito oriundo da cultura organizacional para formar o conhecimento em enfermagem local.

Esse acesso ao conhecimento em enfermagem pode ser usado numa rede de comunicação da prática de enfermagem, por exemplo, o gerenciamento do cuidado através da intranet e Internet. O objetivo é consolidar os diferentes caminhos para prover medidas básicas de custos, qualidade, acesso ao cuidado do paciente e resultado desse cuidado.

6. Goossen WTF, Epping PJMM, Abraham R. Classification systems in nursing: formalizing nursing knowledge and implications for nursing information systems. Meth Inform Med 1996 jan;35(1):59-71.

7. Pan American Health Organization. Building standard-based nursing information systems. Washington (DC): PAHO; 2001.

8. Rodrigues Filho J. The complexity of developing a nursing information system: a brazilian experience. Comp Nurs 2001 may-jun;19(3):98104.

9. Berg M, Bowker GC. The multiple bodies of the medical record towards a sociology of an artefact. Sociol Quarterly1997;38(3):51337. 